DETECTION/DIAGNOSIS

AXILLARY NODES

https://doi.org/10.29289/259453942020V30\$1002

\title{
INTRAOPERATIVE EVALUATION OF SENTINEL LYMPH NODE IN THE ERA OF ACOSOG Z0011
}

Vanessa Monteiro Sanvido¹, Simone Elias¹, Gil Facina¹, Afonso Celso Pinto Nazário

${ }^{1}$ Escola Paulista de Medicina, Universidade Federal de São Paulo - São Paulo (SP), Brazil.

Introduction: Intraoperative evaluation of sentinel lymph node was routinely conducted to assess the presence or absence of metastasis and decide, during the same surgical procedure, whether to perform an axillary dissection, which would avoid a second surgical procedure. However, with the current recommendations for conservative axillary treatment, the role of the intraoperative assessment is questionable. Results of the American College of Surgeons Oncology Group Z0011 (ACOSOG Z0011) randomized trial allow skipping the axillary lymph node dissection in patients with two positive axillary sentinel lymph nodes treated with conservative surgery, which would also allow not performing the intraoperative evaluation of sentinel lymph node because the result would not change the surgical approach. However, the rate of axillary re-excision was not described for cases of ACOSOG Z0011 exclusion criteria after definite anatomopathological results. Objectives: To assess the rate of axillary retreatment in patients submitted to conservative breast surgery in the era of ACOSOG Z0011. Method: This is a retrospective cohort study of patients who had invasive breast carcinoma up to $5 \mathrm{~cm}$, clinically negative axilla, and underwent conservative breast surgery and sentinel lymph node dissection from February 2008 to December 2018. Results: We evaluated 415 patients - 318 (76.7\%) with negative sentinel lymph node, and 97 (23.3\%) with positive. Among positive cases, 56 (57.8\%) were treated with sentinel lymph node biopsy, and 41 (42.2\%) were submitted to axillary lymph node dissection. Intraoperative evaluation occurred in $90.2 \%$ of cases prior to the publication of ACOSOG Z0011, decreasing to 30.8\% after publication ( $\mathrm{p}<0.00001)$. The rate of surgical re-excision due to ACOSOG Z0011 exclusion criteria was only 3.7\%. The main causes were the presence of metastasis in three or more axillary lymph nodes or capsular extravasation. Conclusions: Intraoperative evaluation of sentinel lymph node substantially decreased in patients with early-stage breast carcinoma treated with conservative surgery and sentinel lymph node biopsy after implementing the axillary treatment proposed in the ACOSOG Z0011 guidelines, and the rate of axillary surgical retreatment due to exclusion criteria was minimal. 